\title{
Standardization of Fungal Allergens
}

\author{
Robert K. Bush and John W. Yunginger
}

Aerobiologic sampling studies show that fungal spores have a world-wide distribution, and IgE-mediated sensitivity to fungal allergens is a cause of allergic disease. The diagnosis of fungal hypersensitivity is in part predicated upon the demonstration of binding of $\operatorname{IgE}$ to antigens contained in extracts prepared from the fungi.

In 1980, 281 mold extracts were commercially available in the USA. ${ }^{1}$ These fungal extracts are variable and complex in their constituents. To the clinician faced with the task of diagnosing and treating patients with fungal allergy, the lack of readily available standardized extracts is a major problem. Fortunately, the prospects for significant advances in this area appear bright. In the past few years techniques have been developed that permit adequate study of the allergenic composition and content of such extracts. The isolation and purification of allergens from fungal extracts have further aided the development of standardized allergenic extracts. Efforts are underway by the International Union of Immunologic Societies (IUIS) to develop international reference standards that meet World Health Organization (WHO) requirements. These international reference standards include fungal allergens such as Alternaria and Cladosporium. Three commercially available fungal extract preparations are now licensed as standardized extracts in the United States. Reports of the therapeutic efficacy of standard fungal extracts have begun to appear, and additional studies are in progress.

\section{Epidemiology}

For a comprehensive review of the epidemiology of fungal sensitivity, the reader is referred to several articles. ${ }^{2-4}$ In the natural environment man is exposed to more than 100 species of airborne or dust-bound microfungi. ${ }^{5}$ Fungal spore counts frequently exceed pollen counts in the atmosphere by 1000 -fold. Several genera are considered to be universal dominants in aerosampling surveys. These include Cladosporium, Alternaria, Fusarium, Drechslera (Helminthosporium), and several yeasts. Indoor concentrations of fungi generally depend on the outdoor concentrations. However, certain genera, such

From the Section of Allergy and Clinical Immunology, Department of Medicine, University of Wisconsin, and the William S. Middleton V. A. Hospital, Madison, Wisconsin; and the Allergic Disease Research Laboratory, the Mayo Clinic, Rochester, Minnesota.

Send correspondence and requests for reprints to Robert K. Bush, MD, Section of Allergy and Clinical Immunology, Department of Medicine, University of Wisconsin, 600 Highland Avenue, Madison, WI 53792. 
as Aspergillus and Penicillium, are found indoors on a regular basis and their concentration is not dependent on the outdoor concentration. ${ }^{3}$ A larger number of fungi are recognized as aeroallergens (Table 1). In the United States, Alternaria is a major allergen on the east coast, in the Upper Midwest, and in southern California. ${ }^{6}$ Cladosporium is the most common airborne fungal spore in the United States. Although it does not appear to be as potent an allergen as Alternaria, several species are important allergens, including $C$ herbarum and $C$ cladosporoides. Cladosporium is found in most of the temperate zones of the world.

Allergic reactions attributable to mold sensitivity have long been recognized. In 1726, Sir John Floyer ${ }^{7}$ noted asthma in patients who had just visited a wine cellar. In 1873, Blackley ${ }^{8}$ suggested that Chaetomium and Penicillium were associated with asthma attacks. In 1924, van Leeuwen ${ }^{9}$ noted the relationship of climate to asthma and found a correlation between the appearance of mold spores in the atmosphere and attacks of asthma. At the same time, Cadham $^{10}$ reported the first documented case of asthma due to wheat rust. Over the next 10 years, case reports appeared attributing the source of mold allergy to the home or to occupational settings. In the late 1930s, Prince et $\mathrm{al}^{11}$ and Feinberg ${ }^{12}$ reported that outdoor air was a significant source of mold

Table 1. Genera of Fungi Associated With Allergy in the Literature

\begin{tabular}{|c|c|c|}
\hline Absidia & Drechslera & Papularia \\
\hline Agaricus $^{a}$ & Epicoccum $^{a}$ & Penicillium $^{a}$ \\
\hline Alternaria $^{a}$ & Epidermophyton & Phytophtora \\
\hline Ankistrodesmus & Erysiphe $^{a}$ & Piptoporus \\
\hline Armillaria $^{a}$ & Eurotium & Pleurotus \\
\hline Arthrinium & Fomes & Podaxis \\
\hline Aspergillus ${ }^{a}$ & Fugus & Polyporus \\
\hline Aureobasidium ${ }^{a}$ & Fuligo & Polystictus \\
\hline Boletus & Fusarium ${ }^{a}$ & Puccinia $^{a}$ \\
\hline Botrytis ${ }^{a}$ & Ganoderma $^{a}$ & Rhizopus $^{a}$ \\
\hline Bracteacoccus & Geotrichum & Rhodotorula ${ }^{a}$ \\
\hline Candida $a^{a}$ & Gliocladium & Saccharomyces ${ }^{a}$ \\
\hline Cantharellus & Graphium & Scopulariopsis \\
\hline Cephalosporium & Hormidium & Serpula ${ }^{a}$ \\
\hline Chaetomium $^{a}$ & Hypholoma ${ }^{a}$ & Sphaerotheca \\
\hline Chlorophyllum & Leptosphaera & Spondylocladium \\
\hline Cladosporium $^{a}$ & Lycogala & Sporobolomyces ${ }^{a}$ \\
\hline Claviceps & Lycoperdales genera & Sporotrichum ${ }^{a}$ \\
\hline Conosporium & Macrosporium & Stemonitis \\
\hline Coprinus ${ }^{a}$ & Malessezia & Stemphylium ${ }^{a}$ \\
\hline Cryptococcus ${ }^{a}$ & Microsphaera & Stereum \\
\hline Cryptostroma & Mucor ${ }^{a}$ & Tilletiopsis $^{a}$ \\
\hline Curoularia & Phoma $^{a}$ & Trichoderma \\
\hline Dacrymyces & Mycogone & Trichophyton ${ }^{a}$ \\
\hline Daldinia & Neochloris & Trichothecium \\
\hline Debaryomyces & Neurospora ${ }^{a}$ & Ustilago $^{a}$ \\
\hline Dicoccum & Nigrospora & Verticillium \\
\hline Didymella ${ }^{a}$ & Paecilomyces ${ }^{a}$ & Xylaria \\
\hline
\end{tabular}

Source: Adapted from Koivikkoa: Patient exposure to moulds, in Foucard T, Dreborg S (eds): Mould Allergy Workshop. Uppsala, Pharmacia, 1983, p 35.

${ }^{a}$ Both skin test and provocation test reactions have been observed 
spores and demonstrated that many of their patients had positive skin test reactivity to mold extracts.

Initially, mold sensitivity was equated to skin test reactivity; but it was found that many patients with skin test reactivity did not experience typical symptoms at times of high levels of atmospheric fungal spore activity. This may have been due in part to irritant properties of the extracts used in the testing.

The role of mold sensitivity in a variety of allergic diseases was established in provocation studies. By exposing patients to $1 \mathrm{~g}$ Alternaria powder dispersed in a $700-\mathrm{cu} \mathrm{ft}$ room, Harris ${ }^{13}$ provoked asthma and rhinitis symptoms in 10 of 12 patients with positive skin tests to Alternaria who had a history compatible with sensitivity to the fungus. Only 1 of 10 patients with a positive skin test but less clear-cut history of sensitivity developed symptoms upon similar exposures. Pennington ${ }^{14}$ applied cotton swabs that had been rolled over mold cultures to the nasal mucosa of 61 skin test-positive individuals. Twenty-two reacted to a variety of molds to which they had skin test reactivity. Subsequently, more sophisticated challenge studies have repeatedly demonstrated reactivity to fungal extracts in patients with positive skin tests. Both immediate hypersensitivity and late phase allergic reactions have been induced by provocation studies. ${ }^{15}$ It has recently been demonstrated that inhalation of either Alternaria or Penicillium spores in quantities comparable with those encountered by natural exposure can induce both immediate and late asthma in sensitive persons. ${ }^{16}$

Despite the numerous studies implicating mold sensitivity in the pathogenesis of allergic diseases, the prevalence of mold sensitivity has not been established. In patients with asthma and rhinitis, the reported frequency of positive skin test reactions to fungal allergens varies from $4 \%$ in Scandinavian studies to more than $80 \%$ in certain studies in the United States. ${ }^{2}$ Whereas skin test reactivity indicates the presence of $\mathrm{IgE}$ antibodies to fungal allergens, the correlation between symptoms and skin test reactivity may be only about $50 \%$. The potency of materials used for skin testing significantly influences the results of studies of the prevalence of mold sensitivity. For example, positive skin prick test reactions in patients with histories suggestive of sensitivity to $C$ herbarum varied from 12.5 to $86 \%$ in the same population, depending on the extract used for testing. ${ }^{17}$

Although fungal sensitivity is well recognized, it is not always possible to establish a correlation between mold spore counts in the atmosphere and the presence of allergic symptoms. Bruce et $\mathrm{al}^{18}$ were able to correlate autumnal asthma symptoms with Alternaria spore counts in only 15 of 29 subjects who had demonstrable IgE sensitivity to this fungus. In a study at the University of Wisconsin, only 1 of 20 Alternaria-sensitive subjects demonstrated a positive correlation between allergic symptoms and Alternaria spore counts. ${ }^{19}$

The introduction of aerosampling devices and techniques that can quantitate the amount of allergen in the atmosphere (as opposed to morphologic identification and quantitation of fungal spores by microscopy) may shed further light on this problem. Agarwal et $\mathrm{al}^{20}$ was able to detect Alt-I (the major allergen of Alternaria) in the atmosphere. This correlated well with mold spore counts and with the total Alternaria allergen activity in the atmosphere. 
Since allergenic activity may be borne on particles other than spores, such studies may enhance the measurable correlation between allergen exposure and clinical symptoms.

Environmental surveys have suggested new avenues of approach to the study of fungal allergy. Salvaggio et $\mathrm{al}^{21}$ reported high asthma admission rates to hospitals in New Orleans, which appeared to be associated with high mold spore counts-particularly of small spores, such as those of Basidiomycetes. Since Basidiomycetes are widely distributed and their spores are among the most common found in the atmosphere, investigations are being conducted on their role in allergic disease. Santilli et $\mathrm{al}^{22}$ demonstrated that in 32 ragweedsensitive subjects, 65 to $75 \%$ reacted to skin testing with spore extracts of 3 Basidiomyces species. This was further confirmed by the demonstration of positive radioallergosorbent tests (RAST) to the spore extracts, as well as IgE binding to precipitates in crossed-radioimmunoassay (CRIE) autoradiographs.

Finally, the physician needs to be aware of the possibility of sensitization to fungal materials, not only from natural outdoor exposure but also from indoor exposure. Modest numbers of undisturbed house plants contribute minimally to aeroallergen prevalence in homes. ${ }^{23}$ However, under greenhouse conditions, where large numbers of plants are grown indoors, abundant fungal growth can occur and this can become airborne when the plants are disturbed. Cold air vaporizers also can generate high concentrations of fungal spores, ${ }^{24}$ and marijuana smoking may lead to development of fungal hypersensitivity reactions. ${ }^{25}$

Physicians also must consider occupational exposure as an important source of fungal sensitivity. Positive skin tests and bronchial challenges to Alternaria and Aspergillus occur in patients with baker's asthma, ${ }^{26}$ and there is evidence to suggest that fungal allergens play a role in the etiology of cotton dust sensitivity. ${ }^{27}$

\section{History of Standardization of Fungal Extracts}

Blackley introduced skin testing to the diagnosis of allergic disease in 1873. With the introduction of immunotherapy in $1911,{ }^{28}$ the need for a measure of allergen extract potency was recognized. Noon introduced the Noon unit, ${ }^{28}$ which was equal to the amount of allergen extracted from $1 \mu \mathrm{g}$ pollen. In the following years, allergen extracts were defined on a weight per volume basis (wt/vol), a practice that has persisted to the present day. In the 1930s, it was recognized that most allergens were proteins or glycoproteins. This led to introduction of the protein nitrogen unit (PNU), ${ }^{29}$ which is equivalent to 0.01 $\mu \mathrm{g}$ protein. This unit also has persisted to the present time. However, studies have repeatedly demonstrated that allergen potency is not closely associated with either of these measurements. ${ }^{30}$

Technical advances in the past several decades have improved our ability to quantitate the amount of allergen in various extracts. Notable advances include the discovery of IgE antibody, the ability to measure specific IgE antibodies in serum by the RAST, the RAST inhibition assay, and the isolation and purification of allergens. These techniques have now been applied to fungal allergens. Yunginger et $\mathrm{al}^{30}$ used endpoint skin test titration, RAST 
inhibition, and direct RAST to compare the relative in vivo and in vitro potencies of 12 commercially available Alternaria extracts. While all the extracts carried the same content of PNU or equivalent $w t / v o l$ units on their labels, they differed 300 -fold on endpoint skin testing and 200 -fold by RAST inhibition. Vijay et al $^{31}$ studied batch-to-batch variations in Alternaria extracts from 2 companies. The direct RAST showed little discrimination among the extracts. However, RAST inhibition revealed a 40 -fold variation in potency in batchto-batch products from the same company and a 55-fold difference between extracts prepared by the 2 individual companies. In addition, there were marked differences in the biochemical compositions of the products from the 2 companies.

Studies with the most concentrated commercially available $C$ herbarum extracts showed that their potency was insufficient for the diagnosis of most patients, but that the diagnosis could be established in patients with suspected histories when skin testing and provocation tests with highly purified, biologically standardized, freeze-dried allergen preparations of $C$ herbarum were used. ${ }^{32}$ Batch-to-batch variations in allergens in Mucor racemosus and Aspergillus fumigatus preparations have been reported. ${ }^{33}$ Agarwal et al ${ }^{34}$ studied 8 commercially available Alternaria extracts by isoelectric focusing (IEF), crossedimmunoelectrophoresis (CIE), CRIE, RAST inhibition, and immunoassays for content of Alt-I. Since there were differences in the rank order of allergen potency as measured by the various assays, the authors suggested that the optimal method for mold allergen standardization involves a combination of techniques.

Nationwide efforts in the United States led to the standardization of short ragweed extracts in 1982. Since that time it has been required that all lots of short-ragweed extracts in the United States have a minimum antigen-E content of $135 \mu \mathrm{g} / \mathrm{mL}$ in a $1: 10(\mathrm{wt} / \mathrm{vol})$ extract and that their antigen-E content appear on the label. Individual manufacturers in the United States have introduced standardization into their fungal extract products. Three preparations are available at the time of this writing (Alternaria alternata, 3M Diagnostics, Mountainview, CA; and $A$ alternata and Ustilago spore extracts, Optimal Inc., Bridgeport, CT).

For the past several years, the IUIS has brought together clinicians, scientists, manufacturers, and regulatory agencies in an attempt to develop internationally recognized standards of reference for fungal allergen extracts. These references are not intended to be models of extract preparation by the manufacturers, but rather to establish a potent extract of known composition to which other extracts can be compared. In 1982, the Alternaria Working Group was established. Subsequently, 5 manufacturers submitted preparations for selection as the reference material. One of these products has been extensively evaluated in an international cooperative study involving 22 laboratories throughout the world. Pending the outcome of ongoing stability tests, it is anticipated that this international reference will soon be accepted by WHO. Future studies on standardized fungal extracts by the IUIS will include Cladosporium. Continued efforts should lead to production of quality extracts of known potency that physicians can apply to the diagnosis and treatment of patients. 


\section{Extract Preparation}

Many factors contribute to the marked batch-to-batch variability of fungal extracts. Among these are accurate identification of the source material; biologic characteristics of the fungi after repeated subculture; use of mycelia, spores, or culture filtrate; conditions under which the fungi are grown and extracts are prepared (which often are similar to those used to prepare pollen extracts and may not be suitable for fungi); and stability of the extracts once they are prepared.

\section{Source Material}

Fungi may mutate, producing morphologically variant forms (Fig. 1). As fungal taxonomy is based upon microscopic appearance, particularly of the spores, disagreements may arise among mycologists. ${ }^{35}$ This problem was encountered in the preparation of an international reference extract of Alternaria. ${ }^{36} \mathrm{Six}$ extracts were submitted to the IUIS for evaluation as an international Alternaria reference extract standard. Fifteen strains, independently evaluated by an

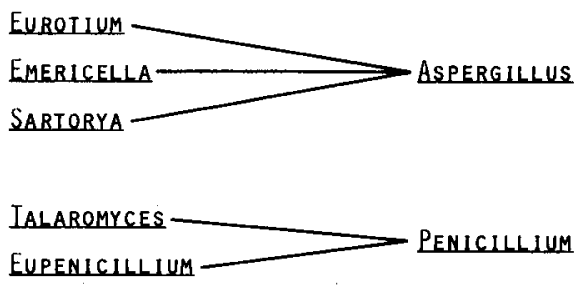

NECTRLA
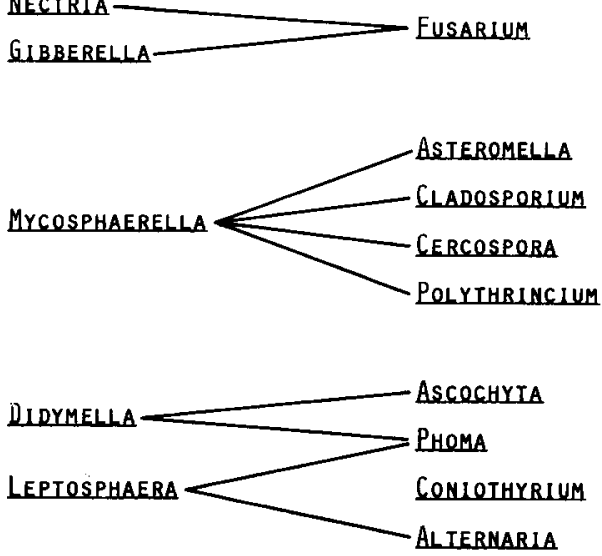

PLEOSPORA

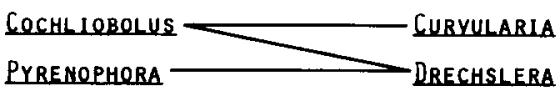

Figure 1. Selected sexual/asexual relationships in the Ascomycetes. All spore types are commonly airborne. (FSN) Adapted, with permission from Burge $H A$ : Fungus allergens. Clin Rev Allergy 3:321, 1985. 
expert in fungal taxonomy, were used to prepare these 6 extracts. Of 5 strains accessioned by the American Type Culture Collection, all were confirmed as being Alternaria. Of 5 strains accessioned through a European repository, 4 were Alternaria and 1 was identified as Ulocladium. Of the 5 strains not accessioned by any national repository, 3 were Alternaria, 1 Ulocladium, and 1 a mixture of Paecilomyces and an unidentified red yeast. One strain was found to be parasitized. Because disagreement exists even among experts, manufacturers may mislabel extracts; not through deliberate misrepresentation or carelessness, but as a reflection of changing fungal taxonomy. For example, Helminthosporum maydis is now considered to be a species of Drechslera, and $H$ interseminatum as a species of Curvularia.

Simply labeling extracts as Alternaria is insufficient. Of 10 manufacturers in the United States, 8 use $A$ alternata, 1 uses $A$ brassicola, and 1 uses an unspecified strain. Furthermore, the allergenicity of 14 strains of $A$ alternata accessioned into the American Type Culture Collection has not been established. Skin testing with Alternaria has demonstrated differences among species, with $A$ tenuissima being the most potent in 1 study. ${ }^{37}$ As a practical and reasonable strategy in standardizing fungal extracts, a reference preparation probably should contain pooled extracts obtained from a number of similar strains grown independently.

Once a particular organism has been chosen, there is the question of whether spores, mycelia, or culture filtrate should be used in preparing the extract. Currently, most commercial extracts are from mycelia and contain little or no spore material. ${ }^{6}$ Several studies indicate that spores differ allergenically from mycelia. ${ }^{38-40}$ Spore preparations may be more active by skin testing and RAST inhibition, ${ }^{41}$ but some investigators ${ }^{42}$ have found that higher spore content did not increase the activity of Alt-I; the major antigen of Alternaria. While spores are the most important source of allergen exposure for most individuals, it is difficult to obtain pure spore preparations at a reasonable cost for commercial extract preparation. It also should be remembered that patients inhale constituents other than spores, such as mycelial fragments that would not be recognizable microscopically and particulate matter to which allergens may be bound. Aukrust ${ }^{35}$ found that Alt-I activity was 3 times higher in mycelial than in spore preparations of Alternaria. Spore preparations were more heterogeneous and contained more allergens than preparations from mycelia. It is therefore suggested that reference materials and commerical extracts contain both spore and mycelial constitutents. While culture filtrates do contain allergenic materials, they often lack sufficient quantities of allergens, and the presence of various enzymes in the culture filtrate may lead to degradation of allergens. Therefore, culture filtrates are probably not a suitable source for preparation of standardized extracts.

\section{Culture Medium}

Many variables affect the production of allergens by fungi. Factors such as glucose supply, oxygen, light and dark cycles, temperature, and $\mathrm{pH}$ may be important in sporulation and thus may affect allergenic composition. Several 
studies suggest that chemically defined media will support the growth of fungi while preserving allergenic activity. ${ }^{43,44}$ Therefore it is recommended that synthetic media be used whenever possible to culture fungi to provide standardized growth conditions and to prevent the introduction of potential allergens of nonfungal origin.

\section{Growth Time}

The optimal length of time fungi should be grown in culture before allergens are harvested remains to be clarified. Kaufman et al $^{45}$ studied the release of IgE-binding antigens of Aspergillus into culture filtrates. There is an early growth phase lasting approximately 10 days, during which IgE-binding antigens appear in the culture filtrate. This is followed by a drop in IgE binding during the second phase, days 10 to 20 , and an increase in the third phase when cells are lysed (approximately 20 to 40 days). It also was noted that different IgE-binding antigens appeared not only during the first and third phases, but also during the same phase when Aspergillus was grown under the same conditions of different occasions. Kroutil et a ${ }^{46}$ examined the appearance of IgE-binding allergens in culture filtrates and mycelial extracts of Alternaria when cultures were harvested after 1, 2, and 3 weeks. They found that the total protein content of the mycelia increased from week to week and the fewest number of proteins were identified by sodium dodecyl sulphate-polyacrylamide gel electrophoresis (SDS-PAGE) at 1 week. However, the greatest IgE binding occurred in extracts prepared from mycelia harvested at 1 week. Furthermore, more allergens were identified by CRIE in the 1- and 2-week mycelial extracts. Culture filtrates, conversely, showed an inverse relationship in allergenic activity, with more allergens observed-in the 2 - and 3 -week culture filtrates than in the 1-week filtrate material. Detailed studies of various fungi will be necessary to establish the ideal culture time for each fungus.

\section{Extraction Process}

Several variables enter into the extraction process. Excessively long extraction times at room temperature or above can lead to destruction of allergens by hydrolytic enzymes in the extract. ${ }^{6}$ Studies on the effect of extraction time on allergen release from Aspergillus spp ${ }^{47}$ indicated that RAST-binding compounds are released within the first 20 minutes of the extraction process. Similar results were obtained with Alternaria. ${ }^{48}$ Powdered mycelia extracted at $\mathrm{pH} 8.0$ at $4^{\circ} \mathrm{C}$ for 15 minutes, 60 minutes, 6 hours, and 24 hours showed increasing IgE binding up to 6 hours by direct RAST. Similarly, the allergens identified by CRIE were maximal at 6 hours and diminished by 24 hours. The authors concluded that allergens are rapidly eluted from mycelia of Alternaria, and extraction times beyond 6 hours may alter the allergenic composition.

The $\mathrm{pH}$ of the extracting buffer also may affect the composition of the allergenic extract. As the $\mathrm{pH}$ of nasal secretions in active rhinitis is 8.0 to 8.5 , extracting the raw material with buffers in this $\mathrm{pH}$ range may produce the most clinically relevant allergens. 


\section{Assessment of Allergenic Activity}

No single available immunochemical assay will properly evaluate the allergenicity of extracts prepared even under standardized conditions. Li et al ${ }^{49}$ evaluated 6 Aspergillus preparations by a variety of immunochemical and physiochemical methods. Like Agarwal et $\mathrm{al}^{34}$ they found that the rank order of antigen and allergen content differed considerably with the method used. Therefore it is suggested that a variety of immunochemical assays are required to effectively evaluate fungal extracts, and comparison of these extracts with a reference material may provide a basis for their standardization.

\section{Stability of Extracts}

No systematic studies evaluating the stability of fungal extracts have been conducted. However, lyophilized Alternaria and Cladosporium extracts that had been stored at room temperature for 15 years compared favorably with freshly prepared extracts when evaluated by skin prick testing. ${ }^{50}$ The addition of $50 \%$ glycerine will help stabilize extracts for skin testing. The stability of extracts used for immunotherapy has yet to be established.

\section{Characterization of Allergens}

Because of the great variability of fungal extracts, their ultimate standardization will depend upon isolation and purification of the individual allergens they contain. Several investigators have isolated and characterized fungal allergens (Table 2).

Currently, the best characterized allergen is Alt-I, derived from Alternaria alternata. ${ }^{51}$ Crude $A$ alternata extract was fractionated by ammonium sulfate precipitation, anion exchange chromatography, flat-bed preparative IEF, and gel filtration. The isolated material contained appreciable amounts of carbohydrate, in keeping with studies showing that removal of $96 \%$ of extractable nitrogen from Alternaria extracts could be achieved without loss of skin test reactivity. ${ }^{52}$ This material had an apparent molecular weight of between 25,000 and 50,000 and an isoelectric point between 4.0 and 4.5. Further evaluation

Table 2. Purified Fungal Allergen Fractions

\begin{tabular}{llccc}
\hline \multicolumn{1}{c}{ Species } & $\begin{array}{c}\text { Allergenic } \\
\text { fraction }\end{array}$ & $\begin{array}{c}\text { Approximate } \\
\text { MW }\end{array}$ & pI & Reference \\
\hline Alternaria tenuis & Alt-I & $25,000-50,000$ & $4.0-4.5$ & 51 \\
A iridis & Ag-I & 60,000 & 4.0 & 53 \\
A alternata & Ag-8 & $35,000-40,000$ & $4.3-4.65$ & 54 \\
A sp & Basic peptide & 9000 & $9.5-9.8$ & 60 \\
Cladosporium herbarum & Ag-32 & 13,000 & $3.7-4.4$ & 67 \\
Aspergillus fumigatus & Ag-54 & 25,000 & 5.0 & 67 \\
\hline
\end{tabular}

Source: Modified from Yunginger JW: Fungal allergens, in Kerr JW, Ganderton MA (eds): Proceedings of Invited Symposia. XI International Congress of Allergology and Clinical Immunology. London, The MacMillan Press, Ltd., 1983 p 539.

$\mathrm{MW}=$ molecular weight; $\mathrm{pI}=$ the $\mathrm{ph}$ of a solution containing a salute at its isoelectric point 
revealed that Alt-I was heterogeneous by thin-layer IEF and polyacrylamide gel electrophoresis. Additional fractionation by preparative polyacrylamide gel electrophoresis showed 5 fractions, 3 of which had similar amino-acid compositions.

Nyholm et $\mathrm{al}^{53}$ found slight biochemical differences between Alt-I and a major allergen, termed antigen 1 (Ag-1), obtained from a different strain of $A$ alternata. By tandem CIE and CRIE, however, these allergens appeared to be identical. The approximate molecular weight of this antigen was 60,000 , and its isoelectric point was 4.0. Radioallergosorbent test inhibition showed parallel inhibition lines, suggesting immunological identity of these components. Further investigations ${ }^{54}$ resulted in the identification of another major antigen, Ag-8, which was partially purified by diafiltration, gel filtration, and preparative IEF. Its molecular weight was between 35,000 and 40,000 , and subsequent evaluation showed it to be a variant of Alt-I. It has been estimated that Alt-I comprises only a small fraction of the total dry weight of $A$ alternata. In the richest extracts examined to date, it comprises only $0.7 \%$ of the dry weight. $^{53}$

The biologic activity of Alt-I has been studied. ${ }^{55} \mathrm{IgE}$ binding was found in sera of 21 of 25 asthmatics sensitive to Alternaria; wheal and flare reactions occurred at levels of nanograms per milliliter in 16 of 25 patients. Bronchial challenges in 11 of 16 individuals using 0.6 to $60 \mu \mathrm{g} / \mathrm{mL}$ of Alt-I produced a greater than $20 \%$ drop in $\mathrm{FEV}_{1}$, and histamine release from leukocytes obtained from sensitive individuals was demonstrated. In further studies, ${ }^{56} 100$ unselected patients reactive to crude Alternaria were skin tested to Alt-I and $83 \%$ reacted. Therefore, Alt-I is a major allergen of Alternaria. Monoclonal antibodies to a subfraction of Alt-I have been prepared. ${ }^{57}$ Development of similar monoclonal antibodies will undoubtedly be of great assistance in the standardization of Alternaria extracts. There also is evidence that suggests Alt$\mathrm{I}$ is the major allergen in Stemphylium ${ }^{58}$ and Ulocladium. ${ }^{59}$

Other investigators have identified additional Alternaria allergens. Budd et $\mathrm{al}^{60}$ obtained an allergenic fraction from an unidentified Alternaria species after water extraction, dialyzation, and preparative flat-bed IEF. This material had an estimated molecular weight of 9000 and an isoelectric point between 9.5 and 9.8. It gave positive skin tests in 18 of 20 patients reactive to crude Alternaria extract. Other studies ${ }^{6}$ confirmed the existence of allergenic activity in basic fractions of Alternaria spore and mycelial extracts.

Hoffman et $\mathrm{al}^{61}$ examined the allergenic characteristic of spore preparations containing 90 to $96 \%$ spores and mycelium preparations containing < $1 \%$ spores. By CIE they found 40 antigens in spore preparations, 8 of which were unique to the spore. A spore-specific allergen was isolated, which had an isoelectric point between 6.2 and 6.3. Alt-I activity was found in both spore and mycelial extracts. Skin testing revealed that the vast majority of patients reacted to both spore and mycelial preparations.

Vijay et $a^{62}$ fractionated $A$ alternata and $A$ solani by passing dialyzed extracts over a gel-filtration column, followed by ion exchange chromatography. Allergenic activity eluted from the gel-filtration column had a molecular weight between 15,000 and 30,000. These studies indicated that the 2 species share common allergenic determinants. 
In a preliminary report, Bush et $\mathrm{al}^{63}$ fractionated an $A$ alternata strain differing from the one reported by Yunginger et al ${ }^{51}$ Following similar separation techniques, they identified a fraction with a molecular weight of approximately 18,600 and isoelectric point of 4.2 . This material had partial but not complete identity with Alt-I. It was capable of producing significant histamine release from leukocytes of 5 Alternaria-sensitive subjects in vitro. This may indicate some strain differences in A alternata.

Further studies of allergens in Alternaria have been conducted using CIE and CRIE. Lowenstein ${ }^{64}$ found 18 separate allergens in a crude Alternaria preparation. This included the major allergen Ag-1, which was subsequently found to be identical with Alt-I. Bush et al, ${ }^{65}$ using a different Alternaria strain, identified 22 antigens by CIE. Three allergens were identified in the extract, of which antigen 3 appeared to be a major allergen, since $100 \%$ of 35 patients studied showed IgE binding to it. It was not determined if this antigen corresponded to Alt-I.

Kroutil and Bush ${ }^{66}$ separated a crude Alternaria allergen extract by SDSPAGE and transferred the proteins to nitrocellulose by the Western blotting technique. Incubation of the nitrocellulose strips with human sera, followed by incubation with radiolabeled anti-IgE and autoradiography, disclosed IgE binding to 2 allergens with molecular weights $>66,000,1$ at 66,000 , and another at 29,000 .

In addition to allergens from Alternaria, 2 major allergens have been isolated from Cladosporium. ${ }^{67}$ Purified allergens were obtained from a Cladosporium preparation by diaflow filtration, gel filtration, and preparative flat-bed IEF. Previous studies using CRIE had shown antigen 32 to be a major allergen and another fraction, antigen 54, to be a potent binder of IgE. Following the fractionation procedures described above, antigen 32 was isolated and found to be a heterogeneous protein with multiple bands at isoelectric points of 3.7 , $3.9,4.1$, and 4.4. The molecular weight was estimated to be 13,000 . It was, however, immunologically homogeneous. The other fraction, antigen 54, has been further purified and characterized. ${ }^{68}$ It is a glycoprotein with a molecular weight of approximately 20,000. Neither allergen was cross-reactive as determined by passive transfer skin testing. Isolates of $C$ herbarum, when analyzed by CIE and CRIE, showed complex patterns. Crossed-immunoelectrophoresis revealed up to 60 precipitates in the extracts. One major, 10 intermediate, and 25 minor allergens were identified. ${ }^{69}$ Allergen contents of 10 isolates of $C$ herbarum were analyzed and found to vary from 0 to $100 \%$ relative to a reference. ${ }^{69}$

A preliminary report of the purification of a major Aspergillus fumigatus allergen has appeared. ${ }^{70}$ This allergen, $\mathrm{Ag}-3$, was prepared by ammonium sulfate precipitation of an $A$ fumigatus culture filtrate. This was followed by gel filtration on Sephacryl 200. The allergen was shown to be a major inhibitor of the RAST. It was heat labile, with a molecular weight of 24,000 , and did not bind to concanavalin $A$. Wallenbeck et $\mathrm{al}^{71}$ conducted immunoelectrophoretic studies with crude $A$ fumigatus extracts. Using a 10-strain mixture extract and hyperimmune antisera, they found 44 precipitates by CIE. Two antigens, antigens 10 and 40 , were determined to be major allergens. There were 10 intermediate allergens and 6 minor allergens. When the strains used 
in the extract were investigated individually, they were found to vary in the quantities of the 4 most important allergens. By gel filtration, Piechura et al $^{72}$ isolated an antigen with a molecular weight of approximately 150,000 from a mycelial preparation. This gave positive immediate skin tests in approximately $50 \%$ of patients with allergic bronchopulmonary aspergillosis. Kauffman et $\mathrm{al}^{40}$ compared the allergenic content of spore preparations, mycelial preparations, and culture filtrates of $A$ fumigatus. They found that disrupted spore antigens did not cross-react with either mycelial or culture filtrate allergens. Only minimal IgE binding could be demonstrated to intact spores since the allergens were secreted into the medium spontaneously. Further investigations leading to the isolation of relevant allergens of Aspergillus are needed.

Finally, steps are being undertaken to identify the major allergic constituents of the Basidiomycetes. Lehrer and Salvaggio ${ }^{73}$ examined 82 patients with possible mold allergy by skin testing to extracts prepared from 6 basidiomycete species. They found the range of positive skin tests to be between 9.7 and $14.6 \%$ of 82 patients. Crossed-immunoelectrophoresis and CRIE studies were conducted on 3 species, Xylobolus frustalatus, Dacrymyces delequescens, and Pleurotus ostreatus. All species showed several antigenic components as well as several allergens, the most reactive being $P$ ostreatus, which contained 13 antigens in a combined mycelial and spore extract. Further studies by the same group ${ }^{74}$ with a spore extract of $P$ ostreatus showed 27 precipitates by $\mathrm{CIE}$. The antigens were found in spore, mycelium, or cap extracts. However, unique spore antigens also were identified, of which 5 were allergens.

Despite the progress being made in the isolation and purification of fungal allergens, much work is needed to quantitate these important allergens in fungal extract preparations used in the diagnosis and treatment of patients.

\section{Cross-Reactivity of Allergens}

The term cross-reactivity refers to the antigenic (IgG) or allergenic (IgE) determinants on molecules from different fungal variants. Historically, skin testing with fungal allergens reveal broad patterns of reactivity. ${ }^{75}$ However, other reports indicate that sensitivity may be species-specific in some instances. ${ }^{76}$ The development of immunologic techniques for studying crossreactivity, such as immunoprecipitation techniques and RAST inhibition, has aided our understanding of this phenomenon but has not completely clarified the situation. Because of the large number of fungal variants found in nature, their taxonomic classification into genera, species, and strains does not account for their immunologic reactivity in human beings. In some instances there is little or no cross-reactivity with IgE antibodies between different genera. Aukrust ${ }^{77}$ found that a crude Alternaria extract gave only weak precipitates with a potent rabbit antiserum against Cladosporium herbarum; and CRIE showed no antigenic cross-reactivity between $C$ herbarum and 2 basidiomycetes, Cantharellus cibarius and Coprinus comatus. Similarly, Karr et $\mathrm{al}^{78}$ were unable to demonstrate cross-reactivity between Alternaria allergens and 3 species of Aspergillus by RAST inhibition. In other cases, there may be extensive cross-reactivity among genera. Agarwal et $\mathrm{al}^{58}$ demonstrated that 
Alternaria and Stemphylium extracts contain multiple shared allergenic and antigenic determinants, through the use of RAST inhibition, immunoelectrophoresis, and immunodiffusion studies with rabbit antisera. In fact, the major allergenic determinant of Stemphylium was Alt-I; and Stemphylium actually showed higher Alt-I activity by double-antibody radioimmunoassays for the antigen than did Alternaria extracts themselves. Similar studies conducted with Ulocladium ${ }^{59}$ also demonstrated that Alt-I was the major allergenic component. Hoffman and Kozak ${ }^{79}$ demonstrated extensive cross-reactivity among Alternaria, Drechslera (i.e., Helminthosporum), Spondylocladium, Stemphylium, and Curvularia. These cross-reactive genera contained an antigen that appears to be distinct from the Alt-I described by Yunginger. Surprisingly, Stachybotrys appears to cross-react with the form genera Alternaria, Stemphylium, and Curvularia. $^{4}$

Among species of the same genus, allergenic similarities may exist. For example, $A$ tenuus, $A$ alternata, and $A$ solani appear to possess common allergenic determinants. ${ }^{62}$ Both shared and unique antigens may occur in different species of the same genus. Karr et $\mathrm{al}^{78}$ examined the antigenic relationships among Aspergillus fumigatus, A glaucus, and $A$ flavus, using RAST inhibition. Each species produced significant but only partial inhibition of RAST by each of the other species analyzed. The data suggested that both shared and unique antigens are present in these 3 species. Using fused rocket and immunoelectrophoresis, cross-reactivity among several strains of Aspergillus was demonstrated. ${ }^{80}$ Nonetheless, in the genus Aspergillus, there appear to be common antigens within the fumigatus and niger groups, which are antigenically distinct from the versicolor, nidulans, and glaucus groups. ${ }^{4}$ Radioallergosorbent inhibition and skin testing revealed unique allergenic properties in 4 Alternaria species (tenuissima, brassicae, alternata, and longissima). ${ }^{37}$ Finally, strains of fungi may show allergenic similarities as well as differences. Shumacher et $\mathrm{al}^{81}$ noted wide biochemical variation in 7 isolates of $A$ alternata; however, precipitin studies indicated extensive cross-reactivity. Isolation of major allergens from $A$ alternata (Alt-I, $\mathrm{Ag}-1$, and $\mathrm{Ag-8}$ ) that are immunologically identical suggests marked similarities among strains. However, the content of Alt-I in these strains varies widely. Similarly pronounced immunologic and biochemical differences in 10 strains of $C$ herbarum have been demonstrated. ${ }^{69}$

In summary, while cross-reactivity appears to occur across broad taxonomic groups, further research is needed to establish the importance and degree of IgE cross-reactivity. Patients with fungal allergy often display parallel and independent multiple sensitivity to a variety of fungi.

\section{Clinical Data From Studies Using Characterized Mold Extracts}

\section{Alternaria}

There is a paucity of clinical data concerning the diagnostic and therapeutic efficacy or safety of well-characterized or standardized Alternaria extracts. The biologic activity of Alt-I, an important Alternaria allergen fraction, has been 
demonstrated at nanogram permilliliter concentrations by skin testing of $\mathrm{Al}$ ternaria-sensitive individuals and in vitro induction of leukocyte histamine release using cells from the same persons. ${ }^{55}$ In an ongoing double-blind, longterm study of Alternaria immunotherapy conducted at Mayo Clinic, 30 Alternaria-sensitive persons, primarily asthmatics, were assigned to injection treatment with either purified Alt-I, a crude Alternaria extract with an equal Alt-I content, or a caramelized histamine placebo solution. Positive bronchial provocation tests with both Alternaria preparations were induced at microgram concentrations of ALT-1 in these study patients. A preliminary report of the findings has appeared. ${ }^{82}$ Acquisition of additional clinical data should be greatly facilitated by development of an international standard Alternaria extract. A candidate standard extract has been selected ${ }^{36}$ and tested in an international multicenter collaborative trial. ${ }^{83}$ If WHO accepts the final working group report on this standard, investigators and commercial supplies will be able to use this standard extract to assign a unitage of allergenic potency to their Alternaria extracts.

\section{Cladosporium}

A group of Scandinavian investigators ${ }^{84}$ has reported a double-blind, placebocontrolled trial of Cladosporium herbarum immunotherapy involving 30 asthmatic children. The lyophilized immunizing extract had been partially purified by ultrafiltration and Sephadex G-100 gel filtration and standardized in Biological Units (BU) by skin prick tests in sensitive individuals. In this method of standardization, used in the Nordic countries, the size of the skin test wheal produced by the allergenic extract is compared with the size of the skin test wheal produced by a histamine chloride solution $(1 \mathrm{mg} / \mathrm{mL})$ in a population of sensitive patients. ${ }^{85}$ Extracts that produce a mean wheal size equal to that of the histamine solution are assigned a potency of $1000 \mathrm{BU} / \mathrm{mL}$.

The children were skin tested and underwent conjunctival and bronchial provocation testing before and 5 to 7 months after initiation of immunotherapy. They maintained symptom and medication score diaries and recorded peak expiratory flow rates twice daily during the 10-week Cladosporium season (August to October). The Cladosporium extracts were reconstituted in saline containing $0.03 \%$ numan serum albumin and administered in a clustered dosage regimen, beginning with a dose of $10 \mathrm{BU}$ and building toward a target maintenance dose of $100,000 \mathrm{BU}$. When the maximum tolerated or maintenance dose was achieved, the extract diluent was changed to $0.2 \%$ aluminum hydroxide and $0.4 \%$ phenol in saline to achieve a depot effect. The placebo injection material contained caramelized histamine.

Only 5 of the 16 Cladosporium-treated children could not achieve the targeted $100,000 \mathrm{BU}$ dose. There were 45 generalized reactions (chiefly asthma, involving $81 \%$ of the 16 children) and 4 large local reactions ( $>10-\mathrm{cm}$ diameter). No systemic or large local reactions were encountered in the placebo-treated group. The total medication and symptom scores over the 10-week scoring period did not differ significantly in the 2 groups, but the medication scores of the active treatment group were significantly lower during the 2 weeks with the highest Cladosporium spore counts. The daily peak expiratory flow 
rates did not differ significantly in the 2 groups. Both the conjunctival and bronchial provocation tests demonstrated a significant decrease in reactivity to Cladosporium in the active treatment group, but no statistically significant decrease in Cladosporium skin test reactivity was seen. The authors speculated that the high incidence of systemic reactions encountered during therapy resulted from continual bronchial inflammation induced by sensitivity to coseasonal or perennial allergens to which the children also were sensitive. They further speculated that the clinical efficacy of immunotherapy would have been greater had patients sensitive only to Cladosporium been enrolled, but such patients are rare.

In the same study, Cladosporium-treated patients showed statistically significant decreases in specific IgE antibody and increases in specific IgG antibody. ${ }^{86}$ Crossed-radioimmunoelectrophoresis experiments showed that the increase in IgG antibodies was directed to several antigens, particularly Ag12 and Ag-26, using the reference numbering system of the authors. ${ }^{87}$ However, there was no correlation between the individual immune response and any clinical parameters showing efficacy, such as changes in bronchial and conjunctival sensitivity and medication scores.

Danish investigators ${ }^{88}$ have used the same Cladosporium herbarum and placebo extracts and dosage regimen to treat 22 Cladosporium-sensitive asthmatic adults. A very high incidence of systemic reactions (mainly asthma) again was noted in the active treatment group, including 4 cases of anaphylaxis that required hospitalization. One year after institution of therapy, the active treatment group showed statistically significant decreases in bronchial and skin test reactivity to Cladosporium, as compared with the placebo-treated group. Neither symptom/medication scores nor specific IgE/IgG antibody levels were reported for these patients.

\section{Summary}

The ability of various fungi to induce human IgE-mediated disease, especially allergic asthma, has been well established. The relative clinical importance of fungal aeroallergens varies considerably in different geographic areas. The magnitude of exposure to fungal aeroallergens can be quantitated more precisely by newer immunochemical assays than by more traditional fungal cultures or quantitation of fungal spores by microscopy. Although the wide variations in biologic potency among commerical mold allergenic extracts have been appreciated for decades, only recently have laboratory technologic advances and the production of international reference extracts permitted the development of standardized fungal extracts of defined potency. Future efforts should be directed toward documenting the expected diagnostic and therapeutic advantages of such standardized fungal extracts.

We are indebted to Carol Steinhart and Marion Bortolon for editorial assistance and manuscript preparation, and to Dr. Sten Dreborg for sharing experimental data in press. Preparation of this manuscript was supported in part by the Veterans Administration. 


\section{References}

1. Seebohm PM: Report on the US panel on review of allergenic extracts, in Brede, Going (eds): Regulatory Control and Standardization of Allergenic Extracts. Stuttgart, Gustave Fischer Verlag, 1980, p 168

2. Lopez M, Salvaggio JE: Mold-sensitive asthma. Clin Rev Allergy 3:183-196, 1985

3. Sneller MR: Mould allergy and climate conditions, in Al-Doory, Domison (eds): Mould Allergy. Philadelphia, Lea and Febiger, 1984, pp 244-266

4. Burge HA: Fungus allergens. Clin Rev Allergy 3:319-329, 1985

5. Weeke E, Bendixen G: Micro-fungal allergy. Allergy 34:131-133, 1979

6. Hoffman DR: Mould allergens, in Al-Doory, Domison (eds): Mould Allergy. Philadelphia, Lea and Febiger, 1984, pp 104-116.

7. Floyer J: Violent asthma after visiting a wine cellar. A Treatise on asthma. London, Innys and Parker, 1745

8. Blackley C: Experimental research on the cause and nature of Catarrhus Aestivus (hay fever or hay asthma). London, Bailliere, Tindall and Cox, 1873 (Revised edition, London, Dawson Pall Mall, 1959)

9. Van Leeuwen WS: Bronchial asthma in relation to climate. Proc R Soc Med 17:19-26, 1924

10. Cadham FT: Asthma due to grain dusts. JAMA 83:27, 1924

11. Prince HE, Selle WA, Morrow MB: Molds in the etiology of asthma and hay fever. Tex State J Med 30:340-344, 1934

12. Feinberg SM: Mold allergy; its importance in asthma and hay fever. Wis Med J 34:254-257, 1935

13. Harris LH: Experimental reproduction of respiratory mold allergy. J Allergy 12:279-289, 1941

14. Pennington ES: A Study of clinical sensitivity to air-borne molds. J Allergy 12:388-404, 1941

15. Metzer WI, Hunninghake GW, Richerson HB: Late asthmatic responses: Inquiry into mechanisms and significance. Clin Rev Allergy 3:145-165, 1985

16. Licorish K, Novey HS, Kozak P, et al: Role of Alternaria and Penicillium spores in the pathogenesis of asthma. J Allergy Clin Immunol 76:819-825, 1985

17. Aas K, Leegaard J, Aukrust L, et al: Immediate-type hypersensitivity to common moulds. A comparison of different diagnostic materials. Allergy 35:443-451, 1980

18. Bruce CA, Norman PS, Rosenthal RR, et al: The role of ragweed pollen in autumnal asthma. J Allergy Clin Immunol 59:449-459, 1977

19. Bush RK, Bedard RM, Petry R, et al: Correlation of allergic symptoms with ragweed pollen and Alternaria spore burdens. NER Allergy Proc 5:151 (Abstr), 1984

20. Agarwal MK, Swanson MC, Reed CE, et al: Immunochemical quantitation of airborne short ragweed, Alternaria, antigen E, and ALT-I allergens: A two-year prospective study. J Allergy Clin Immunol 72:40-45, 1983

21. Salvaggio J, Seabury J, Schoenhardt E: New Orleans asthma V. Relationship between Charity Hospital asthma admission rates, semiquantitative pollen and fungal spore counts, and total particulate aerometric sampling data. J Allergy Clin Immunol 48:96-114, 1971

22. Santilli J Jr, Rockwell WJ, Collins RP, et al: Coprinus micaceus, Fuligo septica, and Alternaria alternata spore allergy in highly ragweed-sensitive subjects. J Allergy Clin Immunol 75:118 (Abstr), 1985

23. Burge HA, Solomon WR, Muilenberg ML: Evaluation of indoor plantings as allergen exposure sources. J Allergy Clin Immunol 70:101-108, 1982

24. Solomon WR: Fungus aerosols arising from cold-mist vaporizers. J Allergy Clin Immunol 54:222-228, 1974

25. Kagen S, Kurup VP, Sohnle PG, et al: Marijuana smoking and fungal sensitization. J Allergy Clin Immunol 71:389-393, 1983

26. Klaustermeyer WB, Bardana EF Jr, Hale C: Pulmonary hypersensitivity to Alternaria and Aspergillus in baker's asthma. Clin Allergy 7:227-233, 1977

27. Butcher BT: Findings suggesting that anti-fungal antibodies may play a role in the etiology of cotton dust induced lung disease, in Foucard, Dreborg (eds): Mould Allergy Workshop. Uppsala, Pharmacia, 1983, pp 91-96 
28. Noon L: Prophylactic inoculation against hay fever. Lancet i:1572-1573, 1911

29. Stull A, Cooke RA, Glidden M: The protein nitrogen unit for standardization of inhalant extracts for diagnosis and treatment of allergic diseases. J Allergy 8:454-456, 1937

30. Yunginger JW, Jones RT, Gleich GJ: Studies on Alternaria allergens II. Measurement of the relative potency of commercial Alternaria extracts by the direct RAST and by RAST inhibition. J Allergy Clin Immunol 58:405-413, 1976

31. Vijay HM, Huang $\mathrm{H}$, Young NM, et al: Studies on Alternaria allergens IV. Comparative biochemical and immunological studies of commercial Alternaria tenuis batches. Int Arch Allergy Appl Immunol 74:256-261, 1984

32. Aas K, Aukrust L: Immediate hypersensitivity responses to fungal agents, in Al-Doory, Domison (eds): Mould Allergy. Philadelphia, Lea and Febiger, 1984, pp 133-146

33. Bousquet J, Guerin B, Furic R, et al: Standardization of allergenic extracts, in Spector (ed): Provocative Challenge Procedures: Bronchial, Oral, Nasal and Exercise, Vol. 1. Boca Raton, FL, CRC Press, 1983, pp 45-96

34. Agarwal MK, Jones RT, Yunginger JW: Immunochemical and physicochemical characterization of commercial Alternaria extracts: A model for standardization of mold allergen extracts. J Allergy Clin Immunol 70:432-436, 1982

35. Aukrust L: Selection of source materials for reference preparations of molds, in Brede, Baer (eds): Regulatory Control and Standardization of Allergenic Extracts. 4th Int. Paul Ehrlich Seminar, 1985 (in press)

36. Helm RM, Aukrust $\mathrm{L}$, Baer $\mathrm{H}$, et al: Production of an international reference standard $\mathrm{Al}$ ternaria extract I. Testing of candidate extracts. Int Arch Allergy Appl Immunol, 1986 (in press)

37. Burge HP, Muilenberg ML, Solomon WR: Alternaria allergens at the species level. J Allergy Clin Immunol 67:69 (Abstr), 1981

38. Solomon WR, Burge HA, Muilenberg L: Allergenic properties of Alternaria spore, mycelial and metabolic extracts. J Allergy Clin Immunol 65:229, (Abstr), 1980

39. Marsh DG, Collins RP, Santilli J Jr: ALT-I and other major Alternaria allergens are spore specific. J Allergy Clin Immunol 73:114 (Abstr), 1984

40. Kauffman HF, van der Heide $S$, Beaumont $F$, et al: The allergenic and antigenic properties of spore extracts of Aspergillus fumigatus: A comparative study of spore extracts with mycelium and culture filtrate extracts. J Allergy Clin Immunol 73:567-573, 1984

41. Santilli J Jr, Rockwell WJ, Collins RP, et al: The standardization of fungal spore extracts. J Allergy Clin Immunol 73:114 (Abstr), 1984

42. Oliver JD, Wahl R, Winter HG, et al: Comparison of Alternaria tenuis extracts prepared from different raw materials. Ann Allergy 55:279 (Abstr), 1985

43. Shafree A: New synthetic medium for the production of Alternaria allergens. Ann Allergy 40:220-222, 1978

44. Bush R, Recht M, Kroutel L, et al: A comparison of extracts obtained from Alternaria grown on synthetic versus organic medium. J Allergy Clin Immunol 75:117 (Abstr), 1985

45. Kauffman HF, van der Heide S, van der Laan S, et al: Standardization of allergenic extracts of Aspergillus fumigatus. Liberation of IgE-binding components during cultivation. Int Arch Allergy Appl Immunol 76:168-173, 1985

46. Kroutil L, Petry R, Bush RK: Allergen production by Alternaria alternata strain 46582 is dependent upon culturing time. J Allergy Clin Immunol 73:113 (Abstr), 1984

47. Rijckaert $G$, Broers JLV: Time dependent release of allergens from some xerophilic fungi. Allergy 35:679-682, 1980

48. Sweeney M, Kroutil L, Recht M, et al: Kinetics of allergen release from Alternaria. J Allergy Clin Immunol 75:118, 1985

49. Li JTC, Swanson MC, Helm RM, et al: The antigenic and allergenic variability of Aspergillus fumigatus extracts. Ann Allergy 55:279 (Abstr), 1985

50. Prince HE, Meyer GH, Morrow MB: Stability of lyophilized Alternaria and Hormodendrum concentrates. Ann Allergy 38:101-103, 1977

51. Yunginger JW, Jones RT, Nesheim ME, et al: Studies on Alternaria allergens III. Isolation of a major allergenic fraction. J. Allergy Clin Immunol 66:138-147, 1980 
52. LeBeau LJ: Allergenic fractions of Alternaria species. Thesis, Chicago, Univ. of Illinois College of Medicine, 1952

53. Nyholm L, Lowenstein H, Yunginger JW: Immunochemical partial identity between two independently identified and isolated major allergens from Alternaria alternata (ALT-1 and Ag-1). J Allergy Clin Immunol 71:461-467, 1983

54. Aukrust L, Almeland T-L, Aas K, et al: Partial purification and characterization of an allergen (Ag-8) and quantification of this allergen in eight strains of Alternaria alternata. Allergy 37(Suppl 1):36-40, 1982

55. Miles RM, Parker JL, Jones RT, et al: Studies on Alternaria allergens IV. Biologic activity of a purified Alternaria fraction (ALT-1). J Allergy Clin Immunol 71:36-39, 1983

56. Yunginger JW: Fungal allergens, in Kerr, Ganderton (eds): Proc Invited Symposium, XI Int Congress of Allergology and Clinical Immunology. London, The MacMillan Press, 1983, PP 537-539

57. Agarwal MK, Helm RM, Hisamatsu K, et al: Monoclonal antibody to ALT-1 and its use in affinity chromatography. J Allergy Clin Immunol 71:160 (Abstr), 1983

58. Agarwal MK, Jones RT, Yunginger JW: Shared allergens and antigenic determinants in Alternaria and Stemphylium extracts. J Allergy Clin Immunol 70:437-444, 1982

59. Gravesen S, Nyholm L: Common major allergens in Alternaria and Ulocladium. Rome, XXX Congress Eur Acad Allergy Clin Immunol (Abstr), 1983

60. Budd TW, Kuo CY, Yoo TJ, et al: Antigens of Alternaria 1. Isolation and partial characterization of a basic peptide allergen. J Allergy Clin Immunol 71:277-282, 1983

61. Hoffman DR, Kozak PP Jr, Gillman SA, et al: Isolation of spore specific allergens from Alternaria. Ann Allergy 46:310-315, 1981

62. Vijay HM, Huang H, Young NM, et al: Studies on Alternaria allergens I. Isolation of allergens from Alternaria tenuis and Alternaria solani. Int Arch Allergy Appl Immunol 60:229-237, 1979

63. Bush R, Kroutil L, Hoopes W: Isolation of an allergenic fraction from Alternaria alternata strain 46582. Fed Proc 42:411 (Abstr), 1983

64. Lowenstein $\mathrm{H}$ : Crossed-radioimmunoelectrophoresis, in Oehling, Glazer, Mathor, Arbesman (eds): Advances in Allergology and Applied Immunology. New York, Pergamon Press, 1980, pp 573-577

65. Bush RK, Voss MJ, Bashirian S: Detection of Alternaria allergens by crossed-radioimmunoelectrophoresis. J Allergy Clin Immunol 71:239-244, 1983

66. Kroutil L, Bush RK: Detection of Alternaria allergens by Western blotting. J Allergy Clin Immunol 75:174 (Abstr), 1985

67. Aukrust L, Borch SM: Partial purification and characterization of two Cladosporium herbarum allergens. Int Arch Allergy Appl Immunol 60:68-79, 1979

68. Sward-Nordmo M, Wold JK, Paulsen BS, et al: Purification and partial characterization of the allergen Ag-54 from Cladosporium herbarum. Int Arch Allergy Appl Immunol 78:249-255, 1985

69. Aukrust L: Allergens in Cladosporium herbarum, in Oehling, Glazer, Mathor, Arbesman (eds): Advances in Allergology and Applied Immunology. New York, Pergamon Press, 1980, pp $475-481$

70. Longbottom JL, Harvey C: Partial characterization of a major allergen (Ag-3) of Aspergillus fumigatus. Ann Allergy 55:280 (Abstr), 1985

71. Wallenbeck I, Aukrust L, Einarsson R: Antigenic variability of different strains of Aspergillus fumigatus. Int Arch Allergy Appl Immunol 73:166-177, 1984

72. Piechura JE, Huang CJ, Cohen SH, et al: Antigens of Aspergillus fumigatus II. Electrophoretic and clinical studies. Immunology 49:657-665, 1983

73. Lehrer SB, Salvaggio JE: Diagnosis of basidiomycetes allergy: The first step, in Foucard, Dreborg (eds): Mould Allergy Workshop. Uppsala, Pharmacia, 1983, pp 81-90

74. Weissman DN, Halmepuro L, Olson J, et al: characterization of a Basidiospore extract by immunoelectrophoretic techniques. Ann Allergy 55:279, 1985

75. Prince HE, Morrow MB: Skin reaction patterns to dematiaceous mold allergens. Ann Allergy 29:535-538, 1971 
76. Rackeman FM, Randolph TG, Guba EF: The specificity of fungus allergy. J Allergy 9:447-453, 1938

77. Aukrust L: Cladosporium herbarum, in Foucard, Dreborg (eds): Mould Allergy Workshop Uppsala, Pharmacia, 1984, pp 61-71

78. Karr RM, Wilson MR, Anicetti VR, et al: An approach to fungal antigen relationships by radioallergosorbent test inhibition. J Allergy Clin Immunol 67:194-198, 1981

79. Hoffman DR, Kozak PD Jr: Shared and specific allergens in mold extracts. J Allergy Clin Immunol 63:213 (Abstr), 1979

80. Kim SJ, Chaparas SD: Characterization of antigens from Aspergillus fumigatus II. Comparison of antigenic relationships of clinically important Aspergilli. Am Rev Respir Dis 120:1297-1303, 1979

81. Shumacher MJ, Farr RS, McClatchy JK, et al: Primary interaction between antibody and components of Alternaria II. Antibodies in sera from normal, allergic and immunoglobulindeficient children. J Allergy Clin Immunol 56:54-63, 1975

82. Carey TL, Miles RM, Jones RT, et al: Controlled double-blind study of immunotherapy for Alternaria allergy. A preliminary report. Clin Res 30:163A, 1982

83. Helm RM, Squillace DL, Aukrust $\mathrm{L}$, et al: Production of an international reference standard Alternaria extract II. International collaborative study. J Allergy Clin Immunol 1986 (in press)

84. Dreborg S, Agrell B, Foucard T, et al: A double-blind multicenter child immunotherapy trial using a purified and standardized Cladosporium herbarum preparation I. Clinical results. Allergy 1986 (in press)

85. Anonymous: Registration of Allergen Preparations. Nordic Guidelines, Pub. No. 7. Uppsala Nordic Council on Medicines, 1982, pp 10-11

86. Dreborg S, Agrell B, Foucard T, et al: Immunotherapy in children with a purified, freezedried Cladosporium herbarum extract II. Specific IgE and IgG results, in Foucard, Dreborg (eds) Mould Allergy Workshop. Uppsala, Pharmacia, 1983, pp 113-117

87. Karlsson R, Einarsson R: A double-blind multicenter immunotherapy trial in children with a purified standardized, and freeze-dried Cladosporium herbarum preparation versus histamine placebo III. IgE- and IgG-CRIE antibody patterns, in Foucard, Dreborg (eds): Mould Allergy Workshop. Uppsala, Pharmacia, 1983, pp 119-126

88. Malling J-H: Immunotherapy with moulds. Preliminary results, in Foucard, Dreborg (eds): Mould Allergy Workshop. Uppsala, Pharmacia, 1983, pp 126-136 